\title{
Justicia normal neoliberal en salud: el caso de la sentencia T-760/08
}

\author{
Daniel Alzate Mora \\ Universidad del Rosario, Bogotá, Colombia
}

\begin{abstract}
RESUMEN
Este texto pretende ofrecer un análisis crítico sobre el reconocimiento que hace la Corte Constitucional del derecho a la salud como fundamental autónomo. Se parte de una presentación del actual sistema de salud, y la intervención de los jueces constitucionales, para luego analizar a profundidad la argumentación de la Corte Constitucional en la sentencia T-760/08. Desde los análisis teóricos de Nancy Fraser y David Harvey se argumenta que la jurisprudencia constitucional articula un discurso de «justicia normal neoliberal en salud».
\end{abstract}

This article intends to offer a critical analysis on how the Colombian Constitutional Court acknowledges the right to health as fundamental and autonomous. The subject starts by presenting the current health care system and showing the intervention of constitutional judges, later there is a deep analysis of the argumentation of the Constitutional Court in sentence T760/08. The theoretical analysis by Nancy Fraser and David Harvey supports the idea in which constitutional jurisprudence formulates a discourse of "normal neoliberal justice in health care".

\section{Palabras clave}

Derecho a la salud, sistema de salud, acción de tutela, Corte Constitucional, justicia normal, neoliberalismo.

\section{Keywords}

Right to health, health care system, writ for the protection of human rights, Normal Justice, neoliberalism.

Introducción

A inicio de los años noventa el gobierno de César Gaviria reformó en Colombia el sistema de salud para superar las falencias del anterior Sistema Nacional de Salud. Esta 


\section{Revista Convergência Crítica \\ Núcleo de Estudos e Pesquisas em Teoria Social - NEPETS

reforma quedó consignada en la ley 100 de 1993, que de forma paralela a la Constitución Política de 1991, instauraron un esquema sustentado en el aseguramiento individual y privado, con estratificaciones en cuanto a los servicios prestados según la capacidad de pago, con la participación del Estado y el mercado ${ }^{1}$.

La puesta en marcha del nuevo sistema de salud ha estado acompañada por un mecanismo ajeno al sistema: la acción de tutela ${ }^{2}$. Este dispositivo jurídico, creado por la Constituyente de 1991, es una acción judicial de carácter subsidiario y residual para el control constitucional de las autoridades públicas y, excepcionalmente, de los particulares ${ }^{3}$. Según la Defensoría del Pueblo, para el 2008 el incremento del número de acciones de tutela en salud fue del 300\% en comparación con las tutelas desde 1999 , año a partir del cual dicha entidad tiene registros ${ }^{4}$.

El uso masivo y constante de la tutela para resolver los conflictos con el sistema de salud, y sus consecuencias asociadas, entre ellas la congestión judicial, no fueron ajenos a la Corte Constitucional, que además de fijar las pautas interpretativas sobre el derecho a la salud, encontró necesario adoptar medidas de mayor alcance para resolver los problemas de justicia con el sistema de salud, como lo hizo en la sentencia T-760 del 2008. Allí la Corte recogió el amplio desarrollo jurisprudencial sobre el derecho a la

\footnotetext{
${ }^{1}$ MónICA URIBE GómEZ, La dinámica de los actores en la implementación de la reforma del sistema de salud en Colombia: exclusiones y resistencias (1995-2003), 8 Revista de Gerencia y Políticas de la Salud (2009).

2 Diego Eduardo López Medina, "Sistema de salud" y "derecho a la salud": Historia de su interrelación en la jurisprudencia constitucional en http://www.acemi.org.co/Docs/juridica/2.1.2.\%20Diego\%20Lopez\%20Sistema\%20de\%20salud\%20y\%20 derecho\%20a\%20la\%20salud.pdf.

${ }^{3}$ CATAlina Botero Marino, La acción de tutela en el ordenamiento constitucional colombiano (Consejo Superior de la Judicatura. 2006).

${ }^{4}$ EqUIPO INVESTIGATIVO DEL PROGRAMA DE SALUd Y SEgURIDAD SOCIAL DE LA DEFENSORÍA DEL PUEBLO, La tutela y el derecho a la salud. Periodo 2006-2008 (Defensoría del Pueblo 2009). 


\section{Revista Convergência Crítica \\ Núcleo de Estudos e Pesquisas em Teoria Social - NEPETS

salud, y dictó una serie de órdenes de carácter general dirigidas a superar las fallas recurrentes identificadas para la garantía del «goce universal, equitativo y efectivo» del derecho a la salud.

Uno de los aspectos considerados como más sobresalientes de esta amplia decisión de la Corte Constitucional es el reconocimiento de la salud como un derecho fundamental autónomo. Sin embargo, hasta el momento los análisis sobre esta decisión de la Corte no se han ocupado de cómo interpretar este cambio constitucional, o establecer cómo avanzó la jurisprudencia de la Corte en al protección del derecho a la salud.

Este texto pretende ofrecer un análisis crítico sobre este reconocimiento que hace la Corte Constitucional, rastrando la construcción de la fundamentabilidad del derecho a la salud. Se parte de una presentación del actual sistema de salud, y la intervención de los jueces constitucionales, para luego analizar a profundidad la argumentación de la Corte Constitucional en la sentencia T-760/08 para darle el carácter de fundamental al derecho a la salud. Partiendo de los análisis teóricos de Nancy Fraser y David Harvey, se argumenta que la jurisprudencia constitucional articula un discurso de «justicia normal neoliberal en salud».

\section{La organización del sistema de salud en Colombia}

Antes de la ley 100 de 1993, en Colombia operaba el Sistema Nacional de Salud encabezado por el Ministerio de la Salud y fragmentado en tres subsistemas: (i) la seguridad social que afiliaba de forma obligatoria a los trabajadores del sector formal y la burocracia del Estado; (ii) el privado compuesto por planes de medicina prepagada 


\section{Revista Convergência Crítica \\ Núcleo de Estudos e Pesquisas em Teoria Social - NEPETS

según la capacidad de pago o el gasto de bolsillo para comprar los servicios requeridos; y (iii) el resto de la población, o era atendida por los hospitales públicos y las entidades sin ánimo de lucro, o no podían acceder a los servicios de salud ${ }^{5}$. Los servicios de salud estaban financiados por las transferencias directas que hacía el Estado hacia los hospitales públicos, modelo conocido como subsidios a la oferta ${ }^{6}$

En su momento diversos estudios evidenciaron que este Sistema Nacional de Salud era altamente segmentado, con enormes inequidades e ineficiente en la utilización de los recursos disponibles ${ }^{7}$, lo cual motivó que a inicio de los años noventa se implementara en Colombia una reforma estructural al sistema de salud ${ }^{8}$.

Este diagnóstico del Sistema Nacional de Salud, así como la orientación de su reforma estructural reflejan el contexto de la crisis de acumulación capitalista que se registró a nivel mundial en la década de los años setenta, en donde se combinaron altas tasas de desempleo y la aceleración de la inflación, que anunciaban un periodo de estancamiento económico a nivel global. Tendencia que fue agravada con la crisis petrolera de 1973

\footnotetext{
${ }^{5}$ DiAnA MARGARITA PINTO MAsís, La competencia regulada y la atención desde la perspectiva del usuario. Evidencia del sistema de salud colombiano, Harvard School of Public Health (2002). FRANCISCO J. YEPES, et al., Luces y sombras de la reforma de la salud en Colombia. Ley 100 de 1993 (Assalud; Universidad del Rosario, Facultad de Economía; Mayol Ediciones. 2010).

${ }^{6}$ SAÚl FRANCO AGUDELO, La salud pública hoy: enfoques y dilemas contemporáneos en salud pública (Universidad Nacional de Colombia. 2002).

${ }^{7}$ IVÁN JARAMILLO, El futuro de la salud en Colombia: ley 100 de 1993 cinco años después. (Fundación Friedrich Ebert de Colombia - Fescol - Fundación Corona, Fundación para la Educación Superior -FES-, Fundación Antonio Restrepo Barco 4 ed. 1999). Pluralismo Estructurado: Hacia un Modelo Innovador para la Reforma de los Sistemas de Salud en América Latina. (1997). FrANCISCO J. YEPES, et al., op. Cit. Nota 7.

${ }^{8}$ Diana Margarita Pinto Masís, op. Cit Nota 7; Francisco J. YePeS, et al., op. Cit. Nota 7. 


\section{Revista Convergência Crítica \\ Núcleo de Estudos e Pesquisas em Teoria Social - NEPETS

producto del embargo de la OPEP, que profundizó la caída de la paridad oro-moneda dispuesta en Bretton Woods 9 .

Ante el desgaste de las políticas keynesianas para hacer frente a esta crisis, las ideas de un reducido grupo de académicos, liderados por Friedrich von Hayek, fueron retomar un enfoque económico neoclásico de la mano invisible del mercado, que para el momento era minoritario, ante el auge de las políticas de intervención de Keynes a nivel mundial. Sin embargo, son estas las premisas que van a dar paso al desmonte del Estado de Bienestar, cuando logran la influencia práctica en diversos ámbitos políticos. Esta corriente va a adquirir mayor fuerza con el premio nobel de economía para Milton Friedman en 1976 profesor de la Universidad de Chicago y su mentor, Hayek quien recibió el mismo premio en $1974^{10}$.

La consolidación definitiva del neoliberalismo como ortodoxia político-económica en la dirección del Estado se dará con el ascenso al poder de Margaret Tatcher en Gran Bretaña y de Ronald Reagan en Estados Unidos en 1979. Ambas administraciones proporcionaron el apoyo político para desmontar los pactos del Estado de Bienestar entre el capital y los trabajadores para dar paso a la reducción de los impuestos que estimularan la creación de empresa, crear entornos de confianza inversionista, privatizar las empresas públicas, desregular los mercado y crear nuevos en aquellas áreas que era exclusivas del Estado, como la seguridad social y la atención sanitaria ${ }^{11}$.

\footnotetext{
${ }^{9}$ DAVID HARVEY, Breve historia del neoliberalismo (Akal S.A. 2007).

${ }^{10}$ Ibíd.

11 Ibíd.
} 


\section{Revista Convergência Crítica \\ Núcleo de Estudos e Pesquisas em Teoria Social - NEPETS

En esta lógica, el Estado como proveedor y centro de la salud pública y los servicios de salud, como lo era Colombia con el anterior Sistema Nacional de Salud, debe ser reemplazado por la privatización y la descentralización ${ }^{12}$, procesos que van a estar jalonados por instituciones multilaterales como el Banco Mundial y el Fondo Monetario Internacional $^{13}$. En el región de América Latina, las reformas a los sistemas de salud han seguido de cerca el modelo de aseguramiento y atención gerenciada de Estados Unidos, exportación producto de la saturación del mercado norteamericano, en donde las compañías aseguradoras encuentran en la región latinoamericana nuevos mercados que permitan el crecimiento y la inversión ${ }^{14}$.

Entre 1985-1993 se desarrolló la reforma estructural al sistema de salud en Colombia. Inició con la ley 10 de 1990 que introdujo la descentralización en el campo de la salud. Posteriormente, la Asamblea Nacional Constituyente de 1991 profundizó la descentralización y estableció la salud como servicio público cuya prestación la haría el sector privado. Este proceso finalizó con la ley 100 de 1993, que organizó el esquema del aseguramiento en regímenes según la capacidad de pago, y las reglas del acceso a la póliza de salud. Este modelo se sustenta a partir de dos elementos: el aseguramiento y la competencia regulada.

\section{El aseguramiento}

\footnotetext{
12 John AKIN, et al., Financing Health Services in Developing Countries: An Agenda for Reform. A World Bank Policy Study (Wold Bank 1987). N. HOMEDES \& A. UGALDE, Why neoliberal health reforms have failed in Latin America, 71.1 Health Policy (2005).

${ }^{13}$ BERNARDO USECHE ALDANA, De la salud pública a la salud privada: una perspectiva global sobre la reforma al Sistema de Salud en Colombia, 6 Revista Palimpsestus (2007/2008).

${ }^{14}$ KAREN STOCKER, et al., The Exportation of Managed Care to Latin America, 340 N Engl J Med (1999). 


\section{Revista Convergência Crítica \\ Núcleo de Estudos e Pesquisas em Teoria Social - NEPETS

Por mandato de la Constitución de 1991, al Estado colombiano le corresponde garantizar el acceso universal al servicio público de salud. La ley 100/93 establece dos regímenes de aseguramiento: el contributivo y el subsidiado, que estratifican a la población de acuerdo con su capacidad de pago. Mientras se alcanza el aseguramiento universal, se prevé que la población que está por fuera de estos regímenes reciban atención como "vinculados al sistema".

En el régimen contributivo se encuentran afiliados los trabajadores con contrato laboral o los independientes que tengan un ingreso de por lo menos un salario mínimo. La financiación proviene de los aportes de las personas que compran este seguro de salud, sin recibir aportes del Estado. La prima que se debe pagar por este seguro corresponde al $12.5 \%$ del ingreso básico del trabajador. Quienes cuentan con un contrato laboral, el empleador aporta el $8.5 \%$ y los empleados el $4 \%$ restante, mientras que los «trabajadores independientes» deben aportar la totalidad del 12.5\%. El 1.5\% del aporte de los afiliados al régimen contributivo se transfiere al régimen subsidiado para ayudar a su financiamiento ${ }^{15}$.

En el régimen subsidiado están todas las personas sin capacidad de pago. Este régimen es producto del cambio estructural en la organización del sistema de salud que reemplaza los subsidios a la oferta, asignación presupuestal a los hospitales, por el subsidio de la demanda, sustentado en la compra de seguros de salud por parte de los diferentes gobiernos locales a las empresas aseguradoras ${ }^{16}$.

\footnotetext{
${ }^{15}$ Yepes, et al., op. Cit. Nota 7.

${ }^{16}$ ALEJANDRO GAVIRIA, et al., Evaluating the impact of health care reform in Colombia: From theory to practice.
} 


\section{Revista Convergência Crítica \\ Núcleo de Estudos e Pesquisas em Teoria Social - NEPETS

\section{La competencia regulada}

El nuevo sistema de salud puso fin al monopolio estatal del aseguramiento y separó los procesos administrativos de la prestación de los servicios de salud. Las Entidades Promotoras de Salud (EPS), en su mayoría de carácter privado, tienen a su cargo la gestión administrativa del aseguramiento. Las EPS conforman redes de servicios de salud, las cuales pueden ser propias o contratadas, conformadas por Instituciones Prestadoras de Salud (IPS) que también pueden ser de carácter público o privado ${ }^{17}$.

Las funciones del Estado son principalmente de regulación, dirigida a corregir las imperfecciones del mercado. Para ello se creó la Comisión de Regulación en Salud $(\mathrm{CRES})^{18}$, conformada por el Ministro de la Protección Social, quién la preside, el Ministro de Hacienda y Crédito Público y cinco comisionados expertos designados por el Presidente de la República.

La CRES tiene a su cargo, entre otras funciones, promover un modelo de competencia regulada a través del Plan Obligatorio de Salud (POS) que es aquel conjunto de actividades, procedimientos, intervenciones, servicios, insumos y medicamentos al que tiene derecho cada persona asegurada. El POS fue concebido como un listado cerrado y fijo de procedimientos y medicamentos, con una serie de exclusiones explícitas que en

\footnotetext{
${ }^{17}$ Yepes, et al., op. Cit. Nota 7. IVÁn JARAMILLO, op. Cit. Nota 9

${ }^{18}$ Esta comisión fue creada con la ley $1122 / 07$, estas mismas funciones estaban a cargo del Consejo Nacional de Seguridad Social en Salud (CNSSS) que estaba compuesto por representantes de diversos actores del sistema, gobierno, aseguradoras, prestadores de servicios, sindicatos, empleadores, pensionados (Yepes, et al., 2010).
} 


\section{Revista Convergência Crítica \\ Núcleo de Estudos e Pesquisas em Teoria Social - NEPETS

ningún caso estarían cubiertas por el seguro médico. Para financiar el POS se diseñó la Unidad de Pago por Capitación (UPC), valor que se le reconoce a las EPS por cada persona que tenga asegurada, y que debe cubrir los costos de atención del POS.

A través de la regulación, el Estado debe fomentar la competencia entre las distintas empresas aseguradoras (EPS) y redes de servicios (IPS) por la calidad y la eficiencia en las gestiones administrativas y la prestación de los servicios de salud, en donde el mercado, como gran telón de fondo, debe optimizar el uso de los recursos disponibles.

Sin embargo, quince años después de la reforma y ante un aumento considerable del gasto en salud los balances sobre los resultados alcanzados son contradictorios. Por una parte los reformistas y el gobierno coinciden en afirmar el importante avance en la cobertura de aseguramiento, el mayor acceso a la primera consulta de la población asegurada y la reducción del gasto de bolsillo.

No obstante, otros estudios señalan que estos resultados positivos deben ser analizados críticamente, por ejemplo el avance en la cobertura del aseguramiento debe examinarse frente al contenido del POS, el cual está estratificado según la capacidad de pago. Por otra parte estudios indican que el aseguramiento no garantiza la calidad de la atención y los servicios. También se ha reportado el deterioro en los indicadores de mortalidad evitable, en el programa de control de la tuberculosis y el incremento en la mortalidad materna y perinatal ${ }^{19}$.

${ }^{19}$ Yepes, et al., op. Cit. Nota 7. 


\section{Revista Convergência Crítica \\ Núcleo de Estudos e Pesquisas em Teoria Social - NEPETS \\ ISSN 2238-9288}

Otros estudios evidencian las diversas barreras de acceso a los servicios de salud, que ha sido uno de los principales motivos por los cuales la ciudadanía ha acudido a la acción de tutela con el fin de que le sea garantizado su derecho a la salud ${ }^{20}$.

\section{La intervención de los jueces constitucionales y la implementación del sistema de}

salud

Desde sus primeras decisiones, la Corte Constitucional ha sostenido una postura amplia respecto a los derechos fundamentales, en contra de las posiciones tradicionales de considerar únicamente como fundamentales, aquellos derechos contenidos en el capítulo I título 2 "de los derechos fundamentales" de la Constitución. Tampoco aceptó la Corte la interpretación restrictiva de la Constitución de reducir los derechos fundamentales al catálogo cerrado de derechos de aplicación inmediata del artículo 85 de la Constitución de $1991^{21}$.

En una de las primeras decisiones de la Corte sobre el derecho a la salud, en la sentencia T-406/92 la Corte revisó la acción de tutela interpuesta por la falta de terminación del alcantarillado a cargo del Estado. La Corte consideró que el saneamiento público y la salud pueden ser derechos fundamentales por su conexidad con principios esenciales dentro de un Estado Social de Derecho tales como la vida y la dignidad humana.

\footnotetext{
${ }^{20}$ Cesar Ernesto Abadía \& Diana G. Oviedo, Bureaucratic Itineraries in Colombia. A theoretical and methodological tool to assess managed-care health care systems, 68 Social Science \& Medicine (2009). ${ }^{21}$ Diego lópez MedinA, op. Cit. Nota 3. Mónica ARBelÁEz RudAs, La protección constitucional del derecho a la salud: la jurisprudencia de la Corte Constitucional colombiana, 14 Derecho y Salud (2006). RODOLFO ARANGO, El derecho a la salud en la jurisprudencia constitucional, in Teoría constitucional y políticas públicas: bases críticas para una discusión (Julio Alexei ed. 2008).
} 


\section{Revista Convergência Crítica \\ Núcleo de Estudos e Pesquisas em Teoria Social - NEPETS

Esta interpretación de la conexidad entre la salud y otros derechos fundamentales abre una línea jurisprudencial múltiples veces reiterada por la Corte, en especial cuando aborda problemas específicos con el sistema de salud: tratamientos médicos a una persona enferma de sida, mora en la prestación de los servicios para enfermedades graves, o la desprotección de grupos específicos como los ancianos o los niños, entre $\operatorname{otros}^{22}$.

La mayoría de conflictos de acceso al sistema de salud giran en torno al POS y los servicios que están por fuera de dicho paquete ${ }^{23}$. La comprensión original del POS excluía a las enfermedades ruinosas o catastróficas, a los enfermos de SIDA, y otro tipo de malestares que no alcanzaba los estándares de costo-eficiencia con que se reglamentó dicho instrumento. $\mathrm{Al}$ respecto la Corte indicó que esta concepción excluyente del POS no es compatible con el derecho a la salud dentro de un Estado Social de Derecho, en especial, cuando se trata de «sujetos de especial protección constitucional», para quienes la salud es un derecho fundamental. En consecuencia, la Corte aplicó la excepción de inconstitucionalidad del POS y ordenó los tratamientos, medicamentos y demás servicios requeridos.

Dado que estos servicios se encuentran por fuera del esquema de financiación (POS UPC), la Corte definió que la carga financiera no podía ser traslada a la EPS que negó el servicio, ya que no se debía a una actuación negligente, sino una falla de todo el sistema. En consecuencia, los costos financieros de los servicios tutelados por fuera del

\footnotetext{
22 Diego lópez Medina, op. Cit. Nota 3. Mónica Arbeláez Rudas, op. Cit. Nota 21. Rodolfo Arango, op. Cit nota 21.

${ }^{23}$ EqUIPO INVESTIGATIVO DEL PROGRAMA DE SALUd Y SEGURIDAD SOCIAL DE LA DEFENSORÍA DEL PUeblo, op. Cit. Nota 5. 


\section{Revista Convergência Crítica \\ Núcleo de Estudos e Pesquisas em Teoria Social - NEPETS

POS debían ser reembolsados a la EPS, confiando en la capacidad de ahorro del sistema de salud a través del Fondo de Solidaridad y Garantía, FOSYGA ${ }^{24}$.

No obstante, el criterio de la conexidad para la tutela del derecho a la salud, resultaba demasiado amplio, generando que cualquier demanda en salud fuera protegida por la Constitución. Esto incrementó las acciones de tutelas y la formación de un POS jurisprudencial, compuesto por aquellos procedimientos que de forma reiterada eran negados por las EPS y que la Corte ordenaba su prestación.

En la sentencia SU-111/97 la Corte Constitucional tecnificó su jurisprudencia y adoptó criterios precisos para determinar cuándo la salud puede ser considerada como un derecho fundamental. En dicha oportunidad la Corte redefinió la interpretación de la conexidad con un criterio más exigente, el del "mínimo vital". De esta forma se pretendió reducir la tutela para aquellos casos en donde la pretensión era absolutamente imprescindible para la conservación de la vida, en palabras de la Corte: "El Estado social de derecho exige esforzarse en la construcción de las condiciones indispensables para asegurar a todos los habitantes del país una vida digna dentro de las posibilidades económicas que estén a su alcance. El fin de potenciar las capacidades de la persona requiere de las autoridades actuar efectivamente para mantener o mejorar el nivel de

\footnotetext{
${ }^{24}$ El Fondo de Solidaridad y Garantía FOSYGA es una cuenta adscrita al Ministerio de la Protección Social manejada por encargo fiduciario, sin personería jurídica ni plante de personal propia, cuyos recursos se destinan a la inversión en salud. Artículo 218 ley 100/93; artículo 1 Decreto 1283/96.

www.fosyga.gov.co 


\section{Revista Convergência Crítica \\ Núcleo de Estudos e Pesquisas em Teoria Social - NEPETS \\ ISSN 2238-9288}

vida, el cual incluye la alimentación, la vivienda, la seguridad social y los escasos medios dinerarios para desenvolverse en sociedad." 25

A partir de la sentencia SU-480/97 la Corte inició a sistematizar el conjunto de condiciones que debía verificar cada juez constitucional para que de forma legítima y moderada se justificara la intervención judicial en el sistema de salud. Para ello la Corte estableció una serie de reglas precisas para acceder al POS jurisprudencial sin afectar el equilibrio financiero del sistema. A su vez se el CNSSS intentó que los conflictos del sistema fueran resueltos internamente, para lo cual expidió la resolución 5061 de 1997 que reglamentó los Comités Técnico Científicos (CTC) con el objeto de "establecer las condiciones y el procedimiento para la prescripción de medicamentos no incluidos en el listado (POS), con criterios de costo-efectividad"26.

Sin embargo, la introducción de los CTC no fue suficiente, y el número de acciones de tutela desde 1999 hasta el año 2006 se profundizó ${ }^{27}$. Esto genera una masificación de la jurisprudencia de la Corte, a través de los “fallos de reiteración”. Los jueces de tutela se limitan a citar la regla fijada por la Corte para acceder a servicios no POS y verificar el cumplimiento de dichas condiciones en el caso concreto.

\footnotetext{
${ }^{25}$ Corte Constitucional de Colombia. Sentencia T-426 de 1992 (M.P. Eduardo Cifuentes Muñoz: junio 24 de 1992) ${ }^{26}$ Diego López MedinA, op. Cit. Nota 3.

${ }^{27}$ El derecho a la salud en perspectiva de derechos humanos y el sistema de inspección, vigilancia y control del Estado colombiano en materia de quejas en salud (Procuraduria General de la Nación 2008).
} 


\section{Revista Convergência Crítica \\ Núcleo de Estudos e Pesquisas em Teoria Social - NEPETS \\ ISSN 2238-9288}

En un texto que "ha llegado a ser canónico" 28 la Corte encuentra que se vulnera al derecho a la salud en conexidad con el mínimo vital cuando en el caso concreto se verifican las siguientes condiciones, regla que se mantiene aún vigente:

“a) que la falta de tratamiento o medicamento excluido por la reglamentación legal o administrativa amenace los derechos fundamentales a la vida, el mínimo vital; b) que no exista un medicamento o tratamiento sustituto dentro del POS, o que de existir, no tenga la misma efectividad; c) que el paciente esté en incapacidad económica de sufragar el costo del medicamento o tratamiento o que no pueda acceder a dicho tratamiento por medio de otro plan o seguro médico; d) que medicamento hubiere sido prescrito por le médico tratante adscrito a la EPS a la que está afiliado el paciente."

\section{La sentencia T-760/08: el derecho a la salud como fundamental autónomo}

La Corte Constitucional a través de su Sala Segunda de Revisión de Acciones de Tutela dictó la sentencia número T-760 de 2008, con ponencia del juez Manuel José Cepeda, quién para la época estaba por dejar su cargo de juez constitucional. El magistrado ponente decidió acumular un conjunto amplio de acciones de tutela, todas relacionadas con barreras en el acceso a los servicios de salud y en su mayoría referido a casos anteriormente resueltos de forma reiterativa por la jurisprudencia constitucional, con el fin de obtener una muestra representativa de los conflictos que se plantean entre los usuarios y el sistema de salud. A su vez estos casos permiten, en criterio de la Corte,

${ }^{28}$ Diego LóPEZ MedinA, op. Cit. Nota 3. 


\section{Revista Convergência Crítica \\ Núcleo de Estudos e Pesquisas em Teoria Social - NEPETS

analizar las distintas "facetas" del derecho a la salud, para así valorar sus efectos, con el fin de garantizar el "goce universal, equitativo y efectivo de este derecho"29.

La Corte recogió la amplia jurisprudencia que ha desarrollado durante los quince años de implementación de la ley 100/93, retomando los elementos que había venido definiendo para delimitar el concepto de derecho fundamental en general y la salud en particular. Advirtió que la sentencia no pretende zanjar el debate en torno a los "derechos fundamentales", no obstante, la jurisprudencia constitucional ha logrado un consenso sobre dos criterios que permiten establecer cuándo se está ante un «derecho fundamental»: “i) que existe un consenso en la dogmática constitucional sobre su fundamentabilidad y ii) que el derecho constitucional esté funcionalmente dirigido a lograr la dignidad humana y sea traducible en un derecho subjetivo."30

La Sala de Revisión encontró en el amplio precedente judicial sobre el derecho a la salud que las anteriores dos condiciones estaban acreditadas, por lo que concluyó que la «estrategia de la conexidad» que había acuñado la jurisprudencia sobre este derecho había caído en desuso dado que de forma cada vez más frecuente se ha reconocido situaciones especiales en donde el derecho a la salud tiene el carácter de fundamental de forma autónoma, y por lo tanto, dicha estrategia para proteger el derecho constitucional a la salud resulta "artificiosa".

\footnotetext{
${ }^{29}$ Corte Constitucional de Colombia. Sentencia T-760 de 2008 (M.P. Manuel José Cepeda Espinosa: julio 31 de 2008)

30 lbíd.
} 


\section{Revista Convergência Crítica \\ Núcleo de Estudos e Pesquisas em Teoria Social - NEPETS

La Corte identificó una serie de situaciones específicas en donde el derecho a la salud adquiere la condición de fundamental autónomo y en caso de amenaza o vulneración puede ser protegido por el juez de tutela. Al respecto la Corte señaló los siguientes eventos:

“i) Acceso a los servicios de salud que se requieran contemplados en los planes obligatorios POS; ii) Los contenidos de la ley 972/05 sobre la atención de enfermedades ruinosas o catastróficas, según el art. 2 de la citada ley; iii) Cuando no se garantiza a todos los seres humanos igualmente dignos y se establecen exclusiones discriminatorias, como el caso de parejas homosexuales cuando la pareja era excluida del régimen de salud; iv) cuando se afecte de forma grave y directa un mínimo vital, por fuera del cual el deterioro orgánico impide una vida normal "31.

No obstante la Corte enfatizó que reconocer la salud como un derecho fundamental no significa que se trate de un derecho absoluto que no esté sujeto a limitaciones, por el contrario, se trata de un derecho que para su protección involucra una diversidad de obligaciones complejas y compromete una variedad de acciones y omisiones del Estado y la sociedad para su protección, garantía y respeto.

En este sentido la Corte indicó que el derecho a la salud, así como los «derechos sociales» en general, tiene distintas facetas, algunas de aplicación inmediata: cuando se reclama una abstención del Estado o de los particulares; o porque la acción estatal no implica el gasto de recursos considerables, por ejemplo brindar información a los

${ }^{31}$ Ibíd. 


\section{Revista Convergência Crítica \\ Núcleo de Estudos e Pesquisas em Teoria Social - NEPETS

pacientes sobre sus derechos; o a pesar de la necesidad de recursos importantes la acción del Estado debe ser inmediata, dada las condiciones de urgencia y necesidad, como ocurre con la atención en salud para todas las personas durante su primer año de vida $^{32}$.

Pero a su vez el derecho a la salud tiene otro tipo de facetas, de carácter prestacional, que demandan acciones de diverso tipo y complejidad por parte del Estado, así como la intervención de las autoridades constitucionalmente establecidas para la garantía progresiva del "goce efectivo" del derecho a la salud. En esta medida, y por mandato constitucional, le corresponde al poder Legislativo definir la política pública que organice y establezca la dirección y control del servicio público de salud.

Esta facetas prestacionales del derecho a la salud deben cumplir con tres requisitos previos, a saber: i) la existencia efectiva de una política pública; ii) que dicha política esté orientada a garantizar el goce efectivo del derecho; iii) que contemple mecanismos de participación de los interesados. Sobre el caso particular del derecho a la salud la Corte encontró que existe una relación armónica entre los postulados constitucionales de donde la Corte deriva el derecho fundamental a la salud y la política pública definida en la ley $100 / 93$

Por otra parte, la Corte indicó que resulta constitucionalmente legítimo no incluir ciertos servicios médicos dentro del POS, dado que el derecho a la salud es limitable y la Constitución no ordena su autorización dado que "su exclusión no desconoce aspectos

32 Ibíd. 


\section{Revista Convergência Crítica \\ Núcleo de Estudos e Pesquisas em Teoria Social - NEPETS

importantes de la salud o de la vida del interesado"33. De esta forma la Corte en su jurisprudencia ha señalado que el derecho a la salud debe estar ajustado a las prioridades que los órganos competentes del sistema dispongan, con el fin de "asignar de manera eficiente los recursos escasos disponibles".

La Corte reseñó un amplio conjunto de decisiones judiciales anteriores que negaron la procedencia de la acción de tutela por tratarse de servicios médicos por fuera del POS que no desconocen el derecho a la salud. Al respecto la Corte enumera los siguientes servicios de salud: i) servicio de salud estéticos, ii) gafas y cirugías de ojos; iii) tratamientos de fertilidad; iv) tratamiento de desintoxicación; v) prótesis; vi) by pass gástrico; vii) servicios de odontología; y viii) alergias, entre otros ${ }^{34}$.

\section{La justicia normal neoliberal en salud}

Los análisis disponibles hasta el momento sobre la sentencia T-760/08 coinciden en resaltar el reconocimiento que hace la Corte Constitucional de la salud como un derecho fundamental autónomo ${ }^{35}$. La Corte no cae en la persistente discusión ideológica sobre

\footnotetext{
33 Ibíd.

34 Ibíd.

${ }^{35}$ A pesar del poco análisis académico sobre la sentencia T-760/08, se destaca el enfoque crítico del profesor Óscar José Dueñas, quien hace un llamado a la prudencia y a no sacralizar algunas sentencias de la Corte Constitucional, como la sentencia que se analiza y el caso del desplazamiento forzado (T025/04) (en la cual también fue ponente el juez Cepeda). Según Dueñas este tipo de sentencias "es conveniente discutirlas, ya que incluyen temas que no se pueden aceptar a la ligera". Ambas decisiones, la T-760/08 y la T-025/04, ameritan un análisis sereno, indica el autor, dado que "admiten algunas críticas" OSCAR JOSÉ DUEÑAS RUíz, Observaciones alrededor de la sentencia T-760/08, in Revisión a la jurisprudencia constitucional en materia de salud: estado de las cosas frente a la sentencia T-760 de 2008 (Villareal María Lucía Torres ed. 2009). Los análisis que han resaltado la importancia de la sentencia T-760/08 se encuentran: RAFAEL MALAGÓN OVIEDO, La sentencia T-760: reseña crítica, in La sentencia T-760: alcances y limitaciones (2010);RAFAEL MALAGÓN OVIEDO, et al., La sentencia T-760: alcances y limitaciones (Universidad Nacional de Colombia. 2010);MANUEL JOSÉ CEPEDA ESPINOSA, et al.,
} 


\section{Revista Convergência Crítica \\ Núcleo de Estudos e Pesquisas em Teoria Social - NEPETS

los derechos de «primera» y «segunda» generación, según la cual son únicamente los derechos civiles y políticos los que tienen el carácter de fundamentales, y no los llamados «derechos sociales», discusión que estuvo presente en el proyecto de acto legislativo número 10 de 2002 que impulsó el Gobierno de Álvaro Uribe a través de su ministro del interior, Fernando Londoño, que intentaba excluir la acción de tutela para la protección de los «derechos sociales», y que han estado presentes para desmontar la tutela para este tipo de derechos ${ }^{36}$.

Se destaca que en la sentencia T-760/08 la Corte Constitucional abandone la doctrina de la conexidad para la protección del derecho a la salud, lo cual permite una importante simplificación en la argumentación de la persona que acude a la acción de tutela para la protección de su derecho a la salud, dado que ya no tendrá que demostrar en su caso concreto, que la amenaza o vulneración de su derecho a la salud presenta una conexidad verificable con otros derechos fundamentales, en especial, con el mínimo vital. $\mathrm{Ni}$ tampoco tendrá que recurrir a la doctrina de los "sujetos de especial protección constitucional", argumentando ser parte de este grupo que ha sido definido por la jurisprudencia de la Corte, para lograr que la salud sea considerada como un derecho fundamental. Actualmente con la nueva jurisprudencia de la Corte, en principio, basta simplemente con alegar la vulneración u amenaza del derecho a la salud, ahora considerado como fundamental autónomo.

Revisión a la jurisprudencia constitucional en materia de salud: estado de las cosas frente a la sentencia T-760 de 2008 (Villareal María Lucía Torres ed., Universidad del Rosario. 2009).

${ }^{36}$ Diego LóPEZ MedinA, op. Cit. Nota 3. 


\section{Revista Convergência Crítica \\ Núcleo de Estudos e Pesquisas em Teoria Social - NEPETS}

Sin embargo, asignarle el carácter de fundamental al derecho a la salud no significa que siempre que sea invocado este derecho, automáticamente se cumpla con el requisito de la "fundamentabilidad" para la procedencia de la acción de tutela, y que este reconocimiento implique que se tiene derecho a cualquier atención o servicio de salud. $\mathrm{Al}$ respecto la Corte ha sido enfática en indicar que los derechos fundamentales no son derechos absolutos, por el contrario, son susceptibles de ser limitados, siempre y cuando se respeten unos límites de razonabilidad y proporcionalidad fijados por la jurisprudencia constitucional.

De lo anterior emerge la pregunta por ¿cuál es el ámbito de protección del derecho fundamental a la salud?, es decir, ¿cuál es el contenido de este derecho y qué tipo de demandas sobre el derecho a la salud pueden ser consideradas como constitucionalmente legítimas? Al respecto, de la revisión jurisprudencial antes reseñada se evidencia que existe un amplio margen de interpretación por parte de la Corte, incluso en la sentencia T-760/08 se afirma explícitamente que: "la Sala de Revisión no entra a establecer en detalle el alcance y contenido del concepto de derecho fundamental, en general, ni con relación al caso concreto de la salud"37, y más adelanta explica que "el ámbito del derecho fundamental a la salud está delimitado por la dogmática constitucional, que reconoce los contenidos garantizados a las personas en virtud del mismo",38.

¿Cómo interpretar esta construcción que ha hecho la Corte Constitucional sobre la salud como un derecho fundamental, así como la definición de su contenido y ámbito de

${ }^{37}$ Corte Constitucional de Colombia. Sentencia T-760 de 2008, op. Cit. Nota 29

38 Ibíd. 


\section{Revista Convergência Crítica \\ Núcleo de Estudos e Pesquisas em Teoria Social - NEPETS

protección? Los análisis jurídicos sobre este aspecto están orientados usualmente a la reconstrucción de la dogmática constitucional, realizando ejercicios de sistematización y esquematización de la profusa jurisprudencia que produce la Corte en materia de salud, que refuerza la construcción formal y racional del derecho ${ }^{39}$. Sin embargo, este tipo de análisis no desarrollan un enfoque crítico sobre la jurisprudencia constitucional.

Para una interpretación que supere los márgenes de la dogmática jurídica tradicional, se utilizaran los planteamientos de Nancy Fraser acerca de las discusiones sobre justicia y la construcción del paradigma de la «justicia normal» ${ }^{40}$, así como los planteamientos de David Harvey acerca del «neoliberalismo» ${ }^{41}$.

Los planteamiento de Nancy Fraser ofrecen una reflexión sobre el "horizonte de la teorización política contemporánea"42, que para la autora está enmarcado en la «condición postsocialista», la cual se encuentra caracterizada por: i) la inexistencia de una alternativa omnicomprensiva como lo fue en su momento el socialismo; ii) las nuevas exigencias de justicia ancladas en el reconocimiento de las diferencias y el abandono de las cuestiones sobre la redistribución; y iii) el surgimiento del neoliberalismo globalizante, que incorpora una agresiva mercantilización y un crecimiento desmesurado de las desigualdades materiales ${ }^{43}$. Si bien Fraser reconoce

\footnotetext{
${ }^{39}$ Rodolfo ARANGo, op. Cit. Nota 21. Mónica Arbeláez Rudas, La protección constitucional del derecho a la salud: la jurisprudencia de la Corte Constitucional colombiana, Derecho y Salud. Julio - Diciembre de 2006. At. 205. MANUEL CALVO GARCíA, Los fundamentos del método jurídico: una revisión crítica (Tecnos. 1994).

${ }^{40}$ NANCY FraSer, Escalas de justicia (Herder. 2008).

${ }^{41}$ DAVID HARVEY, op. Cit. Nota 9.

42 NANCY FRASER, Justicia Social (Uniandes Facultad de Derecho ed., Universidad de los Andes 1997).

${ }^{43}$ NANCY FRASER, lustitia Interrupta. Reflexiones críticas desde la posición "postsocialista" (Siglo del
} Hombre. 1997). 


\section{Revista Convergência Crítica \\ Núcleo de Estudos e Pesquisas em Teoria Social - NEPETS

estar inmersa en este horizonte «postsocialista», su propuesta teórica apunta a desmarcarse de ella para exponer una reflexión crítica sobre la misma.

Nancy Fraser explica que cuando se discute sobre cuestiones de justicia, como por ejemplo la erradicación de la pobreza, las diversas posiciones "comparten un conjunto de supuestos subyacentes" $" 44$ a pesar de las radicales diferencias que puedan darse entre las distintas visiones de la justicia que evoca este debate. Siguiendo con el ejemplo, la erradicación de la pobreza está regularmente enmarcada en el campo económico, especialmente en la distribución de la riqueza. Lo mismo sucede cuando se discute la discriminación de las minorías étnicas. Se comparte de forma habitual que éste tipo de injusticias responden al ámbito cultural, por falta de reconocimiento de las diferencias.

En los anteriores ejemplos sobre formas de injusticia, desigualdades sociales y discriminación, subyace un supuesto de teoría social compartido: las injusticias de la pobreza son un asunto de redistribución, casi siempre económica, mientras que las injusticias culturales son un problema de reconocimiento, relacionado con las identidades. También se suele aceptar, sin que haga parte del debate, quiénes son los actores que pueden hacer reivindicaciones de justicia, que por lo general son individuales, y el tipo de instituciones a las que se deben dirigir, generalmente los Estados territoriales. Estos presupuestos compartidos sobre el marco en que se deben desenvolver las reivindicaciones de justicia constituyen en su conjunto el punto de partida de la discusión y en esa medida, no hacen parte del debate sobre la justicia.

${ }^{44}$ NANCY FRASER, op. Cit nota 40. 


\section{Revista Convergência Crítica \\ Núcleo de Estudos e Pesquisas em Teoria Social - NEPETS \\ ISSN 2238-9288}

A pesar de la dificultad de encontrar discusiones que compartan todos los presupuestos descritos, Fraser argumenta que al estar por fuera del debate y tener algún grado de aceptación, permiten la construcción de un paradigma de «justicia normal», en donde la justicia se discute desde los presupuestos dados, sin salirse de dichos márgenes. En esa medida y retomando los planteamientos de Kuhn de ciencia normal, para Fraser, es posible considerar que también la justicia es normal: "siempre y cuando las discrepancias pública o la desobediencia respecto a sus supuestos constitutivos se mantengan bajo control, ${ }^{, 45}$.

El conjunto de principios subyacentes en la discusión sobre la justicia permiten construir una "gramática discernible" que permita establecer el paradigma de la «justicia normal». Cualquier desviación de dicha gramática será relevada a la esfera privada, o será tachada como «anomalías» que no tienen lugar. Esta expulsión del marco de discusión pretende evitar la acumulación de anormalidades y desviaciones que puedan conducir a una posible desestructuración del discurso. En consecuencia, la gramática de la «justicia normal» permite reconocer cuándo se está ante reclamaciones legítimas de justicia, dado que responden al marco normalizador definido.

El análisis de la «justicia normal» sobre el derecho a la salud se ancla desde la «condición postsocialista» definida por Fraser, y que aquí se hace especial énfasis en el contexto económico y político del llamado «neoliberalismo», del cual se ocupa Harvey $^{46}$. A pesar que el término ha tenido un uso tan extendido, que incluso ha llegado a convertirse en una muletilla sin contenido, Harvey avanza en explicar el contexto

\footnotetext{
${ }^{45}$ NANCY FRASER, op. Cit nota 40.

${ }^{46}$ DAVID HARVEY, op. Cit. Nota 9.
} 


\section{Revista Convergência Crítica \\ Núcleo de Estudos e Pesquisas em Teoria Social - NEPETS

histórico en que surge esta nueva configuración, para lo cual va distinguir entre dos tipos de proyectos distintos: uno utópico y otro político.

Para Harvey, los ideólogos del neoliberalismo plantean un proyecto utópico, en tanto que su apuesta es realizar un diseño teórico que permita reorganizar el capitalismo a escala internacional. Este proyecto, como se indicó antes, inició en un grupo minoritario de académicos, liderado por Hayek y complementado con las doctrinas monetaristas de Milton Friedman, que se sustenta en que la libertad y la dignidad de los individuos sólo pueden ser garantizadas mediante el libre mercado, amenazada por la intervención estatal y la regulación de la economía. La teoría político-económica del «neoliberalismo», en su sentido utópico, plantea que el bienestar del ser humano se alcanza fomentando el libre desarrollo de sus capacidades, y las libertades empresariales, por lo que cualquier restricción debe ser abolida. El Estado se debe limitar a garantizar el marco institucional que sustente el libre mercado y las libertades de comercio de los individuos, o crear nuevos mercados en aquellos sectores que anteriormente estaban a su cargo.

Desde la década de 1970, este proyecto utópico del «neoliberalismo» se va a generalizar a nivel mundial, desatando diversos procesos de desregulación de mercados, privatización de empresas públicas y la reducción del Estado en aquellas áreas de provisión social que en el modelo de Estado de Bienestar se encontraban a su cargo. El primer experimento de este proceso se dio en Chile y posteriormente en Argentina, a partir de la dictaduras que fueron utilizadas como estrategia de choque para introducir 


\section{Revista Convergência Crítica \\ Núcleo de Estudos e Pesquisas em Teoria Social - NEPETS

estas reformas $^{47}$. Pero este experimento en la periferia sería trasladado al centro a finales de los setenta, con el asenso de Regan y Tatcher al poder. También otros Estado tradicionalmente definidos como social-democracias o con Estados de Bienestar sólidos como Nueva Zelanda o Suecia, han ajustado sus políticas y prácticas a las premisas del canon «neoliberal». ${ }^{48}$ A su vez los ideólogos del neoliberalismo ocupan importantes posiciones dentro del ámbito académico, en las instituciones centrales del Estado como Ministerios de Economía o las Bancos Centrales, pero también en las instituciones financieras internacionales como el Banco Mundial, el Fondo Monetario Internacional o el Banco Interamericano de Desarrollo. De modo que el discurso del «neoliberalismo» se ha tornado hegemónico ${ }^{49}$.

Sin embargo, Harvey propone examinar con detalle las prácticas y procesos que permiten la «neoliberalización» que evidencia el proyecto político que está detrás del modelo teórico que construyen los ideólogos del neoliberalismo. Esta perspectiva del «neoliberalismo» como proyecto político, plantea que su pragmática no está sustentada en alcanzar los ideales de libertad y dignidad, sino que por el contrario está orientada a recuperar las condiciones necesarias para la acumulación de capital y el restablecimiento en el poder de las élites económicas. La pretendida pureza de la teoría «neoliberal», sustentada por el paradigma científico de economistas ganadores del premio Nobel, resulta cuestionable ante las contradicciones de su puesta en marcha ${ }^{50}$.

\footnotetext{
${ }^{47}$ NAOMI KLEIN, La doctrina del shock. El auge del capitalismo del desastre (Paidós. 2007).

${ }^{48}$ DAVID HARVEY, op. Cit. Nota 9.

49 DAVID HARVEY, op. Cit. Nota 9.

${ }^{50}$ DAVID HARVEY, op. Cit. Nota 9.
} 


\section{Revista Convergência Crítica \\ Núcleo de Estudos e Pesquisas em Teoria Social - NEPETS

Una de estas contradicciones es el papel del Estado. Para la teoría «neoliberal» éste debe garantizar un marco institucional que permita el libre mercado, o la creación de nuevos en aquellas áreas donde no existían, como es el caso de la seguridad social en salud. Una vez La intervención estatal en los mercados debe ser mínima, ya que no posee toda la información suficiente para su regulación, o porque el Estado puede ser cooptado por intereses minoritarios que actuarían en su propio beneficio. No obstante, la pragmática neoliberal del proceso de acumulación de capital y el restablecimiento de las élites económicas en el poder requiere que paradójicamente e n el Estado disponga de las estructuras militares, policiales y jurídicas para asegurar que los derechos inalienables a la propiedad privada, la obtención de beneficios y el funcionamiento de los mercados sean asegurados, incluso mediante la fuerza. Así, lejos del ideal de un "Estado mínimo" el «neoliberalismo» requiere de un Estado interventor que permita la acumulación y no se oponga al restablecimiento de clase.

A partir de las ideas expuestas sobre la «justicia normal»y el «neoliberalismo», se realiza un análisis crítico del proceso de construcción de la "fundamentabilidad" del derecho a la salud, y se presentan algunas consideraciones acerca del cambio jurisprudencial de la Corte en la sentencia T-760/08.

Como se presentó en el recuento jurisprudencial, las primeras decisiones de la Corte Constitucional privilegiaron una interpretación orientada hacia al carácter de «fundamental» del derecho a la salud, para lo cual elaboró diversas construcciones dogmáticas, en especial la doctrina de la «conexidad». En principio, esta interpretación de la Corte puede ser considerada como «anormal», dado que el contexto está ordenado 


\section{Revista Convergência Crítica \\ Núcleo de Estudos e Pesquisas em Teoria Social - NEPETS

por una gramática distinta, que considera la salud como un derecho aspiracional, de carácter prestacional que debe ser desarrollado por el Legislador. A su vez se sostiene que la prestación de los servicios de salud involucra importantes recursos públicos que no permiten su aplicación inmediata y por lo tanto, no puede ser considerado como fundamental.

También en estas primeras decisiones la Corte sostuvo una postura crítica ante la implementación de las políticas «neoliberales» en salud, por ejemplo sobre la definición del POS. En criterio de la Corte las consideraciones de costo-eficiencia con que se formula el conjunto de servicios de la póliza resultaba incompatible con el derecho constitucional a la salud, dado que la exclusión de ciertos servicios podía afectar de forma grave derechos fundamentales como la vida o la integridad personal, como sucedió en el caso de las personas con VIH-SIDA. Bajo esta interpretación de un criterio amplio de conexidad del derecho a la salud como la dignidad humana, la vida, la integridad personal en el marco del Estado Social de Derecho, la Corte logró desarrollar una justicia «anormal» que cuestionara los presupuestos del sistema de salud y a su vez fuera crítica de las políticas «neoliberales», reducidas a un examen de costo-eficiencia.

Ante el incremento de acciones de tutela sobre el derecho a la salud y las presiones por el creciente activismo de la Corte, la «anormalidad» de concebir la salud como un derecho fundamental por conexidad con criterios amplios, se va a ir reduciendo paulatinamente, para terminar normalizando la justicia en salud de forma funcional al «neoliberalismo». 


\section{Revista Convergência Crítica \\ Núcleo de Estudos e Pesquisas em Teoria Social - NEPETS

La justicia normal neoliberal en salud va a consolidarse en la sentencia T-760/08, al sistematizar sus presupuestos y su forma de funcionamiento. Según esta decisión, existen dos consensos de dogmática constitucional sobre el ámbito del derecho fundamental a la salud: i) cuando de la aplicación de la regulación en salud se deriva un derecho subjetivo; ii) cuando es necesaria la excepción de inconstitucionalidad ante la vulneración o amenaza del derecho a la salud.

El primer supuesto para considerar la salud como derecho fundamental parte de la normativa que rige el derecho a la salud, esto es, la Constitución, el bloque de constitucionalidad, las leyes y demás normas de inferior jerarquía que desarrollen este derecho, sea posible concretar una garantía subjetiva. Por ejemplo, las normas que rigen el POS, establecen una garantía subjetiva, dado que este paquete de servicios debe ser garantizado a toda persona asegurada, según el régimen al que corresponda. La normalización opera al dar por sentada la reglamentación del derecho a la salud, por lo que las reclamaciones deben ajustarse a las reglas del sistema, siguiendo en el ejemplo, su demanda debe ser de algún medicamento, servicio o intervención incluida en el POS. A su vez la justicia de la Corte se alinea con el discurso «neoliberal», en la medida en que no cuestiona la lógica del aseguramiento y su circuito financiero, sin que por le contrario, encuentra que en este caso el derecho a la salud es fundamental, dado que cuando interviene en este tipo de casos encuentra que sus medidas no afecta el equilibrio financiero del sistema, lo que permite que la "fundamentabilidad" de la salud sea fácilmente reconocible. 


\section{Revista Convergência Crítica \\ Núcleo de Estudos e Pesquisas em Teoria Social - NEPETS

El segundo consenso es que pueden existir servicios que están excluidos por la normatividad que desarrolla el derecho a la salud pero que son requeridos con necesidad por comprometer gravemente diversos bienes jurídicos protegidos constitucionalmente como la salud, la dignidad humana o la integridad personal. Este segundo supuesto permite un espacio para una justicia «anormal» que cuestione sus presupuestos, como a su vez oponerse a las lógicas de la «neoliberalización» de la salud. Pero por el contrario, en este caso también se evidencia un reforzamiento por la normalidad y el neoliberalismo.

El acceso a los servicios excluidos del POS está sometido a un estricto escrutinio y control, ejercido por los diversos actores del sistema. Se empieza por el personal médico que debe realizar su ejercicio profesional en diagnósticos a partir de la medicina basada en la evidencia, esto es, el contenido del POS, concebido como un catálogo cerrado y rígido; a su vez las EPS tienden a desincentivar los diagnósticos por fuera del POS, y establecen diversas estrategias para que los servicios médicos se mantengan dentro de dicho marco; en caso de que un médico ordene procedimientos por fuera del POS, se debe pasar a la evaluación del CTC, que con criterios de costo-eficiencia, debe establecer si la EPS autoriza el tratamiento no POS.

Si la negación persiste, se puede acudir a la acción de tutela, previo el agotamiento del anterior trámite administrativo, y el juez constitucional debe realizar un estricto escrutinio de las condiciones que ha definido la Corte Constitucional para acceder a medicamentos o tratamientos excluidos del POS: amenaza o vulneración de derechos fundamentales como la vida, la integridad personal o el mínimo vital; que no exista otro 


\section{Revista Convergência Crítica \\ Núcleo de Estudos e Pesquisas em Teoria Social - NEPETS}

tratamiento dentro del POS que tenga la "misma efectividad"; que la persona no esté en capacidad económica de comprar el medicamento o acceder a él mediante otro esquema de aseguramiento (medicina prepagada o plan complementario); que el médico tratante se encuentre adscrito a la EPS a la que está afiliado el paciente; y constatar que los servicios de salud no estén expresamente excluidos del POS. Después de este riguroso examen el juez constitucional puede conceder el amparo constitucional y además ordenar que el costo de los servicios prestados sean recobrados ante el FOSYGA.

Lo anterior constituyen los requisitos para que una persona pueda acceder a servicios de salud que se encuentren excluidos del POS, que constituyen los «itinerarios burocráticos» ${ }^{51}$, tanto al interior del sistema de salud como de la rama judicial, por el que deben pasar las personas para acceder a este tipo de servicios. Esto se traduce en la normalilzación de la justicia en salud, al establecer cuáles son los parámetros que se deben reunir para que la demanda en salud sea considerada como constitucionalmente legítima. Estos parámetros, tanto los del sistema de salud como los judiciales no hacen parte del debate, y se erigen como los presupuestos desde los cuales se parte para hacer uso de la acción de tutela para la protección del derecho a la salud. Por su parte, el activismo judicial de la Corte en busca de no alterar el equilibrio financiero del sistema, exime a las EPS de cubrir los costos de servicios por fuera del esquema de financiación POS - UPC. Esto garantiza que el esquema no se rompa, brindando seguridad jurídica que permite el cálculo capitalista que requieren las EPS para implementar diversas estrategias de contención de costos, por lo que la Corte incentiva la acumulación de capital de las EPS.

${ }^{51}$ Cesar Ernesto Abadía \& Diana G. Oviedo, op. Cit. Número 20. 


\section{Revista Convergência Crítica \\ Núcleo de Estudos e Pesquisas em Teoria Social - NEPETS

A partir de estos dos consensos sobre el ámbito de protección del derecho fundamental a la salud quedan configurados los presupuestos de la «justicia normal neoliberal» en salud, en la medida en que las reivindicaciones de la salud, cuando se acude a la acción de tutela, deben estar enmarcadas en este contexto, y responder a estos parámetros, ya que las desviaciones van a ser consideradas como «anormales» y por fuera del ámbito de protección del derecho fundamental a la salud. A su vez, la justicia de la Corte Constitucional en materia de salud termina colonizada por el discurso «neoliberal», garantizando los derecho de propiedad privada de las EPS que bajo las fórmulas contractuales están blindadas para no interferir en la acumulación de capital, que la Corte permite con las órdenes de sus sentencias.

De lo anterior se concluye que el reconocimiento de la salud como derecho fundamental que ha sido considerado como un importante avance en la protección de este derecho, tiene un alcance reducido, dado que su contenido y ámbito de protección se limita a: i) las políticas neoliberales en salud a partir de la ley 100/93 y sus desarrollos normativos, orientados por los principios de costo/eficiencia; y ii) a ciertas excepciones que están sometidas a un estricto y riguroso escrutinio caso por caso, en donde sólo ciertos servicios de salud están legítimamente protegidos por la Constitución, siempre y cuando no se afecte el equilibrio financiero del sistema y no haga parte de las exclusiones explícitas del POS.

De lo expuesto ser argumenta que la definición del derecho a la salud como fundamental, siguiendo a Fraser y Harvey, configura un discurso de «justicia normal 
neoliberal en salud» en la medida en que las reivindicaciones del derecho a la salud deben someterse a este marco normalizador para que sean protegidas por la acción de tutela, mientras que esta justicia garantiza la acumulación de capital a los entes privados que intervienen en el mercado de la salud.

\section{Bibliografía}

(2008). El derecho a la salud en perspectiva de derechos humanos y el sistema de inspección, vigilancia y control del Estado colombiano en materia de quejas en salud. Bogotá, Procuraduria General de la Nación

(2009). La tutela y el derecho a la salud. Periodo 2006-2008. Bogotá, Defensoría del Pueblo.

Abadía, C. E. and D. G. Oviedo (2009). "Bureaucratic Itineraries in Colombia. A theoretical and methodological tool to assess managed-care health care systems." Social Science \& Medicine 68(6): 1153-1160.

Akin, J., Birdsall, et al. (1987). Financing Health Services in Developing Countries: An Agenda for Reform. A World Bank Policy Study. Whashington D.C., Wold Bank.

Arango, R. (2008). El derecho a la salud en la jurisprudencia constitucional. Teoría constitucional y políticas públicas: bases críticas para una discusión. J. Alexei. Bogotá, Universidad Externado de Colombia.

Arbeláez, R. M. (2006). "La protección constitucional del derecho a la salud: la jurisprudencia de la Corte Constitucional colombiana." Derecho y Salud 14(2): 205-240.

Botero, M. C. (2006). La acción de tutela en el ordenamiento constitucional colombiano. Bogotá, Consejo Superior de la Judicatura.

Franco, A. S. (2002). La salud pública hoy: enfoques y dilemas contemporáneos en salud pública. Bogotá, Universidad Nacional de Colombia.

Gaviria, A., C. Medina, et al. (2006). Evaluating the impact of health care reform in Colombia: From theory to practice. Bogotá., Universidad de los Andes.

Harvey, D. (2007). Breve historia del neoliberalismo. Madrid, Akal S.A.

Homedes, N. and A. Ugalde (2005). "Neoliberal reforms in health services in 


\section{Núcleo de Estudos e Pesquisas em Teoria Social - NEPETS}

Latin America: A critical view of two cases." Revista Panamericana de Salud Pública 17(3).

Homedes, N. and A. Ugalde (2005). "Why neoliberal health reforms have failed in Latin America." Health Policy 71.1: 83-96.

Jaramillo, I. (1999). El futuro de la salud en Colombia: ley 100 de 1993 cinco años después. Santa Fe de Bogotá, Fundación Friedrich Ebert de Colombia - Fescol - Fundación Corona, Fundación para la Educación Superior -FES-, Fundación Antonio Restrepo Barco.

López Medina, D. (2008). "Sistema de salud" y "derecho a la salud": Historia de su interrelación en la jurisprudencia constitucional. Bogotá, Informe preparado para ACEMI.

Pinto, M. D. M. (2002). "La competencia regulada y la atención desde la perspectiva del usuario. Evidencia del sistema de salud colombiano." Harvard School of Public Health.

Stocker, K., H. Waitzkin, et al. (1999). "The Exportation of Managed Care to Latin America." N Engl J Med 340(14): 1131-1136.

Uribe, G. M. (2009). "La dinámica de los actores en la implementación de la reforma del sistema de salud en Colombia: exclusiones y resistencias (1995-2003)." Revista de Gerencia y Políticas de la Salud 8(17): 44-68.

Useche, A. B. (2007/2008). "De la salud pública a la salud privada: una perspectiva global sobre la reforma al Sistema de Salud en Colombia." Revista Palimpsestus 6: 123-131.

Yepes, J. F., M. Ramírez, et al. (2010). Luces y sombras de la reforma de la salud en Colombia. Ley 100 de 1993. Bogotá, Assalud; Universidad del Rosario, Facultad de Economía; Mayol Ediciones. 